\title{
REBECCA ROGERS ET PASCALE MOLINIER (DIR.), LES FEMMES DANS LE MONDE ACADÉMIQUE. PERSPECTIVES COMPARATIVES Elsa Boulet
}

\author{
La Découverte | «Travail, genre et sociétés »
}

$2018 / 1 \mathrm{n}^{\circ} 39 \mid$ pages 225 à 226

ISSN 1294-6303

ISBN 9782707199683

Article disponible en ligne à l'adresse :

https://www.cairn.info/revue-travail-genre-et-societes-2018-1-page-225.htm

\section{Pour citer cet article :}

Elsa Boulet, « Rebecca Rogers et Pascale Molinier (dir.), Les Femmes dans le monde académique. Perspectives comparatives », Travail, genre et sociétés 2018/1 ( $\left.\mathrm{n}^{\circ} 39\right)$, p. 225-226.

DOI 10.3917/tgs.039.0225

Distribution électronique Cairn.info pour La Découverte.

(C) La Découverte. Tous droits réservés pour tous pays.

La reproduction ou représentation de cet article, notamment par photocopie, n'est autorisée que dans les limites des conditions générales d'utilisation du site ou, le cas échéant, des conditions générales de la licence souscrite par votre établissement. Toute autre reproduction ou représentation, en tout ou partie, sous quelque forme et de quelque manière que ce soit, est interdite sauf accord préalable et écrit de l'éditeur, en dehors des cas prévus par la législation en vigueur en France. Il est précisé que son stockage dans une base de données est également interdit. 


\section{Rebecca Rogers et Pascale Molinier (dir.)}

\section{Les Femmes dans le monde académique. Perspectives comparatives}

\section{Presses universitaires de Rennes, Rennes, 2016, 228 p.}

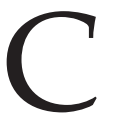

et ouvrage est issu d'un colloque qui a eu lieu en mars 2015 à Paris ${ }^{1}$, et qui a réuni sociologues, historiennes, psychologues et universitaires impliquées dans la promotion de l'égalité femmes-hommes au sein de l'enseignement supérieur et de la recherche (ESR). Il s'organise en trois parties : la première, historique, revient sur l'histoire de quelques "pionnières » (étudiantes puis enseignantes et chercheuses); la seconde présente un panorama international des inégalités de genre au sein de l'enseignement supérieur et de la recherche et propose des analyses de la persistance de ces inégalités ; la troisième partie explore les politiques institutionnelles de promotion de l'égalité entre femmes et hommes, soit en en exposant les présupposés et les angles morts théoriques, soit en se penchant sur la manière dont elles sont appliquées.

Plusieurs problématiques transversales se dégagent de l'ouvrage. D'une part, celle de l'accès au monde académique. Trois contributions traitent de l'accession des premières femmes à des postes d'enseignantes et de chercheuses dans différentes disciplines. Les auteures (Agnès Graceffa, Caroline Barrera et Nathalie Pigeard-Micault) mettent en avant la lenteur de l'intégration des femmes dans le monde académique, avec des étapes souvent longues de "stagnation » à des postes subalternes d'assistantes ou de techniciennes. Dans une perspective contemporaine et comparative, plusieurs chapitres portent sur l'accès, et souvent le non-accès, des femmes aux postes les plus élevés de l'enseignement supérieur et de la recherche, en rappelant la sous-représentation des femmes à ces postes (Nicky Le Feuvre, Marguerite AkossiMvongo et Hassan Guy Roger Tieffi, Danièle Magloire). D'autres contributions analysent la manière dont des politiques institutionnelles tentent de renforcer la présence des femmes à ces postes, des politiques qui soulèvent des questions méthodologiques autour du niveau de l'intervention (suivi individuel, politique au niveau du laboratoire, de la faculté, de l'établissement) et des objectifs poursuivis, ainsi que des interrogations quant aux normes implicites et genrées qui régulent le travail universitaire (Anne-Sophie Godfroy, Farinaz Fassa, Meike Higlemann et Jennifer Niegel). D'autre part, l'ouvrage fournit des informations et des analyses stimulantes sur la question de l'expérience du travail dans l'enseignement supérieur et la recherche, en mettant en avant la façon dont le genre façonne le rapport au travail, pour les enseignantes et les chercheuses mais aussi pour les travailleuses administratives et techniques, plus durement touchées encore par la souffrance au

${ }^{1}$ Colloque

«Les femmes dans le monde académique », du 25 au 27 mars 2015,

Université

Sorbonne

Nouvelle-

Paris 3, Paris

Descartes, Paris

Diderot, Paris

13-Villetaneuse.

Ce colloque a

été organisé

par les

enseignantes-

chercheuses

chargées

de mission égalité de ces

établissements : respectivement Marie-Soledad Rodriguez, Rebecca Rogers, Anne Kupiec et Pascale Molinier. 
${ }^{2}$ Lorraine

Tournyol du Clos et Thomas Le Jeannic, « Les violences faites aux femmes ", Insee Première, $\mathrm{n}^{\circ} 1180$, février 2008.

${ }^{3}$ Discrimination, négation de la légitimité et des compétences, harcèlement : les violences subies par les personnes (et notamment les femmes) racisées en milieu universitaire sont généralement invisibilisées. Une exception notable a été l'atelier « L'expérience des racisées en milieu universitaire : entre résistance, agency et lutte pour la légitimité » au sein du $7^{\mathrm{e}}$ Congrès international des recherches féministes dans la francophonie (août 2015,

Montréal,

Canada). travail (Pascal Barbier et Bernard Fusulier, Alban Jacquemart et François Sarfati, Sophie Lhenry). Enfin, quelques contributions sont consacrées aux femmes et au genre en tant qu'objets scientifiques : Danièle Magloire revient sur la difficile introduction du genre dans l'enseignement en Haïti, tandis que Francine Descarries livre une cartographie et un historique de l'institutionnalisation et la diversification de ces études au Québec et au Canada anglophone.

Ce recueil constitue une source importante pour toutes celles et ceux qui s'intéressent à la division genrée des postes, des tâches et des responsabilités au sein du monde académique. Toutefois, quelques réserves méritent à notre sens d'être formulées. L'ouvrage est fortement structuré autour d'une réflexion sur les femmes enseignantes-chercheuses, au détriment des employées administratives qui constituent pourtant la majorité de la main-d'œuvre féminine de l'enseignement supérieur et de la recherche, à l'exception de l'article très instructif d'Alban Jaquemart et François Sarfati. Le traitement historique de l'accès des femmes à l'enseignement supérieur et la recherche est plutôt une " histoire des grandes femmes » qu'une histoire sociale, qui aurait le mérite de faire apparaître la manière dont les ressources de classe et de capital social de certaines "pionnières " leur ont permis de prendre place dans un monde d'hommes. On peut regretter également que la question des violences sexistes et sexuelles soit absente, alors que le lieu de travail constitue un des endroits où les violences envers les femmes sont les plus importantes (avec le foyer ${ }^{2}$ ) et on sait bien malheureusement que l'enseignement supérieur et la recherche ne font pas exception. Il n'est pas fait mention non plus de la structuration raciale de ce milieu, alors que la sous-représentation voire l'absence totale d'universitaires racisé.e.s est flagrante en France ${ }^{3}$. Enfin, les effets des réformes néolibérales de l'enseignement supérieur et de la recherche sont évoqués mais ils ne sont pas analysés en tant que tels, alors qu'ils constituent à notre avis un élément déterminant dans la permanence, voire l'aggravation, des inégalités de genre : réfléchir et agir sur les inégalités au sein de l'enseignement supérieur et de la recherche passe par une mise en perspective et une remise en cause des politiques éducatives et scientifiques.

\section{Elsa Boulet}

Centre Max Weber, Université Lyon 2, IRIS, EHESS 